\title{
Sistema de vigilancia y control del Reino Nazarí en Granada
}

\author{
Manuel Argüelles Márquez
}

\section{INTRODUCCIÓN}

Hace años iniciamos el estudio de las torres del Reino de Granada, centrándonos en la zona fronteriza situada frente a Alcalá La Real (Argüelles 1987; Salvatierra, Argüelles, Moreno 1989).

El presente texto pretende ser una recopilación de todo el trabajo de prospección realizado durante bastantes años. Además de las zonas fronterizas, donde el número de localizaciones ha aumentado ligeramente, se incluyen numerosos lugares localizados en la Vega'.

Como es bien sabido estas torres pueden dividirse de forma general en dos grupos, las atalayas, con funciones exclusivas de vigilancia y control del territorio apoyando a una gran fortaleza o ciudad, y las torres de alquería, ligadas a una o más localidades, y que servirían como punto de refugio y defensa de la población campesina, cumpliendo funciones hasta cierto punto similares a la de los husun rurales del levante (Guichard 1986) o de la Alpujarra (Cressier 1984; 1992).

No obstante, haciendo abstracción de dicha división y contemplando la distribución de todas las torres y su situación en los cerros, el conjunto da la impresión de configurar varias líneas que pueden leerse de Norte a Sur, ya que en general parecen seguir los cursos de algunos rios o aprovechar pasos naturales, pero que también, además de las que corresponden a la frontera propiamente dicha, parecen formar unas líneas transversales en el interior, con un trazado de Este a Oeste, a modo de cinturones concéntricos en torno a la capital del reino, aunque la articulación de unos $\mathrm{u}$ otros tenga diferentes grados de intensidad, resultado de la diferente densidad del poblamiento. En conjunto formaban un sistema que podía ser utilizado para que mediante señales se comunicasen las novedades producidas desde una a otra, a modo de línea "telegráfica", hasta la misma Alhambra de Granada². Aunque la construcción de las torres se realizase en diferentes momentos, tenemos que resaltar la perfecta distribución de las mismas, resultado de un estudio geográfico y de un perfecto conocimiento del terreno.

A pesar de las guerras internas y de las diferencias existentes dentro del reino, o precisamente a causa de ellas, este sistema sería el más rápido para mantener informado todo el territorio, desde los castillos fronteros hasta tierras situadas en el interior de la provincia de Granada.

\footnotetext{
I Mi agradecimiento por su colaboración a los señores D. Juan Carlos Ureña Coca; D. Alfonso Mazuecos Moraga; D. José Pérez Quesada; D. Fernando Alba y D. Jorge Soria Navarrete.

2 En su día, a una hora convenida y contando con un equipo de colaboradores cada uno con un espejo, distribuidos por los cerros donde se encuentran los restos de las torres, pudimos contemplar desde la Torre de la Vela de La Alhambra, a lo largo del horizonte, los destellos de los espejos, lo que demuestra la importancia de esta línea de torres desde Loja, para mantener informada a la misma ciudad de Granada.
} 


\section{TORRES DE LA FRONTERA}

(Colomera-Moclín-Montefrio)

La línea de fortalezas establecida por los nazaríes frente a Alcalá La Real, y los accesos desde las tierras de Jaén por esta zona, estaba constituida por las de Colomera, Moclín, Íllora y Montefrio. De ellas, la primera quedaba ligeramente escorada hacia el Este, en una zona más montañosa y de dificil acceso. En realidad el núcleo principal estaba formado por las de Moclín y Montefrio, que constituían las alcazabas de las dos localidades principales de la frontera, cada una de ellas protegida por un conjunto de torres, mientras que otras se situaban entre ambos focos, sirviendo además de barrera adelantada a lillora, fortaleza situada entre ambas, pero en posición mas retrasada, y que cerraba la frontera.

\section{ÁREA DE COLOMERA}

\section{I.- La Atalaya (Colomera)}

Situada a 1032 metros de altura, cerca del cortijo El Chopo, a $2 \mathrm{kms}$. de la fortaleza de Colomera en dirección norte. A $3^{\circ} 42^{\prime} 22^{\prime \prime}$ de longitud y $37^{\circ} 23^{\prime} 58^{\prime \prime}$ de latitud. Construida con mampostería, es de planta redonda, maciza, y sólo conserva cinco metros de altura, estando muy deteriorada en la parte norte. Vigilaba las tierras, hacia el norte de Benalúa de las Villas, y al oeste las tierras entre Colomera y Moclín (Lám. I).

\section{ÁREA DE MOCLÍN}

\section{2.- Torre de La Porqueriza (Moclín)}

Situada a $3^{\circ} 47^{\prime} 16^{\prime \prime}$ de longitud y a $37^{\circ} 22^{\prime}$ $4^{\prime \prime}$ de latitud, a $900 \mathrm{~m}$. de altura sobre el nivel del mar, en un lugar estratégico a $2 \mathrm{kms}$. de Tózar y a 3 de Moclín. Es maciza hasta media altura, con una puerta a $6 \mathrm{~m}$. de altura, reforzada con piedra de cantería. Es de planta redonda. Tenía visión directa con las torres de Mingoandrés y con Moclín, controlando los valles orientales próximos a Alcalá la Real. En la parte Oeste del cerro aparece gran cantidad de cerámica hispanomusulmana, monedas y puntas de flecha, el conjunto debió constituir una alquería (Lám. 2).

\section{3.- Torre de Mingoandrés (Moclín)}

Su silueta es visible desde gran distancia en la cima del cerro del mismo nombre, frente al poste que indica el punto $\mathrm{km}$. 410 de la carretera de Granada a Alcalá la Real. Su situación en el mapa corresponde a $3^{\circ} 48^{\prime} 48^{\prime \prime}$ de longitud y a $37^{\circ} 21^{\prime} 20^{\prime \prime}$ de latitud. Con 990 m. sobre el nivel de mar, dominaba todas las torres próximas, controlando una amplia faja de terreno hacia el Norte y el Este, frente a Alcalá la Real (Lám. 3).

\section{4.- Torre de La Solana (Moclín)}

Está situada en una zona que vigilaba los barrancos más próximos a la fortaleza de Moclín. Está a 2 kms. de la fortaleza y a 300 m. de la carretera Moclín-Puerto Lope. A $3^{\circ} 48^{\prime} 9^{\prime \prime}$ de longitud y $37^{\circ} 20^{\prime} 53^{\prime \prime}$ de latitud. Conserva una altura de 6,80 m., de planta redonda, con una habitación en la parte superior.

\section{5.- Alto de la Torrecilla (Moclín)}

Situada sobre un pequeño cerro en el Alto de la Torrecilla a 1.196 m. en la Sierra de Moclín, a poca distancia del punto más elevado de la sierra, el Morrón de Catena. A $3^{\circ} 49^{\prime}$ 05" de longitud y a $37^{\circ} 19^{\prime} 36^{\prime \prime}$ de latitud. Totalmente destruida, en la zona hay materiales cerámicos y ladrillos, así como restos de estructuras de escasa entidad. La zona fue destruida en este punto durante la Guerra Civil por trincheras que cruzan el asentamiento. Tiene visión directa con Moclín y otras torres del sistema.

\section{6.- Torre de la Gallina. (Íllora)}

Situada a $3^{\circ} 50^{\prime} 2^{\prime \prime}$ de longitud y a $37^{\circ} 19^{\prime}$ 23" de latitud, en el Alto de la torrecilla, en la ladera Oeste de la Sierra de Moclín, a pocos metros de distancia de la carretera de Alcalá la Real, en el km. 413 y a 2,5 kms. de la población de Puerto Lope. De construcción maciza y planta redonda. En la parte sudoeste una pequeña puerta conducía a una especie de sótano abovedado. Fue destruída por un rayo en 1970. En torno hay bastante cerámica y restos de construcciones. Aunque el tipo de torre se aproxima a los tipos de atalaya, presenta algunas peculiaridades que la conectan con los tipos de alquería. 


\section{7.- Torre de La Mesa o de La Cuesta. (íllora)}

Situada a 4 kms. de Íllora, a una altura de $926 \mathrm{~m}$. sobre el nivel del mar, frente al cerro de La Laguna, cerca de la Sierra de Madrid, y a $300 \mathrm{~m}$. del cortijo de La Cuesta. A $3^{\circ}$ 50' 5 I" de longitud y a $37^{\circ}$ 18'35" de latitud. Es de planta redonda y construcción maciza, con una altura conservada de 6'90 m. algo deteriorada en su base por la parte Este. Tiene visión directa con la torre de La Gallina y con el Tajo del Sol, y a mayor distancia con la torre de Mingoandrés (Lám. 4).

\section{ÁREA INTERMEDIA}

\section{8.- Torre del Morrón. (íllora)}

Situada a $1.604 \mathrm{~m}$. de altura, a $3^{\circ} 55^{\prime} 34^{\prime \prime}$ de longitud y $37^{\circ} 18^{\prime} 22^{\prime \prime}$ de latitud, es el punto más alto de la Sierra de Parapanda. En la actualidad no quedan restos, ya que en el año 1960 fue destruida para montar el repetidor de TV. Debió ser un punto clave en el sistema de vigilancia por su visión directa de un amplio número de torres (Tocón, La Encantada, las torres de Montefrío, La Mesa, etc.), así como de las tres fortalezas del sistema. Según las noticias existentes, era cilíndrica con una habitación. En el momento de ser destruida se conservaban más de $3 \mathrm{~m}$. de altura.

\section{9.- Torre de Jorbas. (illora)}

En la actualidad es sólo un montón de piedras, con una base cuadrada y 1,70 m. de altura conservada. Se encuentra cerca del cortijo de los Arbitrios, y del que da nombre a la torre, a $300 \mathrm{~m}$. de la carretera de Íllora a Montefrío, en la parte norte de la Sierra de Parapanda, a $3^{\circ}$ 54' 35" de longitud y $37^{\circ} 20^{\prime} 00^{\prime \prime}$ de latitud. En las proximidades aparece abundante cerámica, y se ha localizado una necrópolis destruida en parte por los trabajos agrícolas. Se trata muy posiblemente de una alquería con su torre; por ahora es imposible determinar si se abandonó tras la caída de Alcalá la Real, o continuó habitada. Su posición permite vigilar perfectamente esta última población y su castillo.

\section{ÁREA DE MONTEFRÍO}

10.- La Torrecilla (en el límite Montefrío-IIllora)

Situada en la cota que le da nombre, Loma de la Torrecilla, en la parte Oeste de Sierra de Parapanda, a 4 kms. aproximadamente de la torre de Tocón, y a 2 kms. de la torre del Morrón. La situación de esta torre tan cerca de la del Morrón, probablemente se debió a que la última está en invierno cubierta de nubes. Desde este punto se divisan las torres de Tocón, el Cerro de la Torre - El Amarguillo (V. Mesias), La Encantada y otras dos torres de Montefrío: Los Guzmanes y Torre Quebrada. Con esta torre quedaba establecida una línea entre el grupo de torres más próximo a Montefrío, con Tocón y el grupo de íllora, para enlazar señales y vigilar los puntos oscuros, barrancos y gargantas del arroyo de los Molinos, al oeste de la Sierra de Parapanda. Sus restos fueron desmontados por los labradores y hoy no queda nada más que el nombre, conservado en un cortijo próximo y en la Loma de la Torrecilla. Hay restos romanos en el cercano cortijo Mairena, y un cementerio visigodo en el cortijo Moyano.

\section{II.- Torre Quebrada (Montefrío)}

Situada a $1.222 \mathrm{~m}$. de altura, a 4 kms. en dirección norte de la fortaleza de Montefrío. A $3^{\circ} 57^{\prime} 55^{\prime \prime}$ de longitud y a $37^{\circ}$ $2 l^{\prime} 42^{\prime \prime}$ de latitud. En la actualidad sólo conserva una altura de dos metros, es de planta redonda. Es de suponer que sería una torre de grandes proporciones por la altura que forma el cerro y los materiales acumulados a su alrededor. Es de suponer que su construcción sería de mampostería. Vigilaba las tierras que limitan con Alcalá la Real, Alto de la Peñuela, Venta de los Agramaderos y S. Agreda. Tenía visión directa con la fortaleza y otras torres del sistema: Los Molinos, Los Anillos en el Sur, y La Torrecilla, al Oeste.

\section{2.- Torre del Cortijuelo (Montefrío)}

Situada a 1500 m. de Montefrío, en el Cerro del Cortijuelo, a $3^{\circ} 59^{\prime} 4^{\prime \prime}$ de longitud y $37^{\circ} 20^{\prime} 4^{\prime \prime}$ de latitud. Es maciza, de planta circular, con una altura conservada de unos $6 \mathrm{~m}$. 
Tiene buena visibilidad de la Sierra de la Cazuela y de las lomas próximas a Alcalá la Real. Por la parte Oeste se distinguen las torres de los Anillos y del Morrón (Lám. 5).

\section{3.- Torre de los Anillos (Montefrío)}

Construida en el Alto de los Molinos, a 2 kms. de Montefrío, a $3^{\circ} 58^{\prime} 46^{\prime \prime}$ de longitud y $37^{\circ} 19^{\prime} 0^{\prime \prime}$ de latitud. Es de planta circular, maciza, realizada con piedras de gran tamaño. Conserva unos $3 \mathrm{~m}$. de altura. Controlaba la parte Norte de la Sierra de Parapanda, existiendo visibilidad además de con la torre anterior, con la del Morrón y la de Los Guzmanes (Lám. 6).

\section{4.- Torre de Los Guzmanes (Montefrío)}

Se sitúa a $4 \mathrm{~km}$. de Montefrío, en una elevación a $1.056 \mathrm{~m}$. sobre el nivel del mar, cerca de la carretera que comunica Montefrío con Tocón, a $3^{\circ} 58^{\prime}$ 59" de longitud y $37^{\circ}$ 18' 32" de latitud. Es, como las anteriores, de planta circular y maciza, conservando $5,80 \mathrm{~m}$. de altura, algo deteriorada en su base. Es la torre más aislada de la zona, controlando algunos sectores de la Sierra de Parapanda, y especialmente el Arroyo de los Molinos y el Cerro del Cerrajón. Cabe la posibilidad de que entre esta torre y la de Tocón existiese otra, hoy desaparecida. Se ubicaría en un cerro próximo, denominado de La Torrecilla. Precisamente este punto completa la línea visual entre Montefrío y Tocón, completando algunos ángulos muertos para las otras torres (Lám. 7).

\section{5.- Torre del Hachuelo (Montefrío)}

Situada a $1.136 \mathrm{~m}$. de altura, en el cerro del mismo nombre; $1.10 \mathrm{~m}$. de altura. A $4^{\circ}$ 0 I' 16" de longitud y a $37^{\circ}$ 18' $58^{\prime \prime}$ de latitud. De planta redonda, maciza, realizada en mampostería, su altura es de dos metros, aunque desmoronada. Se distingue perfectamente su planta redonda. Torre principal en el sistema de vigilancia y control por su situación privilegiada, a 3 kms. de la fortaleza, tenía visión directa con la fortaleza de Montefrío, y las torres del entorno de ésta, así como la torre del Morrón, y hacia el sur la torre de la Encantada (Brácana), dando vista a la Vega de Granada y otros puntos oscuros para la fortaleza, en dirección a Algarinejo.

\section{6.- Torre del Sol (Montefrío)}

Situada a $4 \mathrm{kms}$. de la fortaleza de Montefrío, en dirección a Algarinejo, a 907 m. de altura, en el paraje denominado Cortijo de la Torre del Sol. A 4 4' 40" de longitud y a $37^{\circ} 19^{\prime} 28^{\prime \prime}$ de latitud. Conserva una altura de I,40 m. De planta redonda. Construida de mampostería. Controlaba las tierras en dirección a Algarinejo y otras de la Sierra de Chanzas. Tenía visión directa con la fortaleza y torre Quebrada.

\section{ÁREA DE ÍLLORA}

\section{7.- Torre del Charcón (íllora)}

Situada a $100 \mathrm{~m}$. del arroyo del mismo nombre y a $200 \mathrm{~m}$. del casco urbano de íllora, sobre una pequeña elevación que forma pendiente hacia el puente y la carretera de Íllora a Alomartes. A $3^{\circ}$ 53' 6" de longitud y $37^{\circ}$ 17' 6" de latitud. De planta cuadrada, muy derruida, conserva una altura de 1,90 m., con lados de 4,90 m. Parece haber dispuesto de una habitación. Domina el paso del puente del Charcón, así como una serie de barrancos próximos a la fortaleza de Íllora. En los alrededores hay restos de construcciones y abundante cerámica. El conjunto pudo ser una alquería con su torre, en las proximidades de la propia Íllora, formando quizá un barrio de la localidad.

\section{8.- Tajo del Sol (íllora)}

Se encuentra en la Sierra de Madrid, a $1.256 \mathrm{~m}$. de altura sobre el nivel del mar, a 4 $\mathrm{km}$. de distancia de Illora, a $3^{\circ} 52^{\prime} 33^{\prime \prime}$ de longitud y $37^{\circ} 18^{\prime} 17^{\prime \prime}$ de latitud. Esta cota forma una planicie en lo alto del Tajo, desde donde se tenía visión directa de las torres del Morrón y de la Mesa, así como de la de Jorbas, situada a mayor distancia. Se trata de un asentamiento de época nazarí, y quizá anterior, que presenta una muralla de piedras sin argamasa de $150 \mathrm{~m}$. de largo, protegiendo la parte sur de la planicie; en todo el yacimiento aparece abundante cerámica, especialmente en unas cuevas existentes en la parte Este. En las proximidades se encuentra la Fuente Madrid, que desde antiguo abastece de agua a la población de Illora. 


\section{9.- El Fuerte (Íllora)}

Situado a $816 \mathrm{~m}$. de altura sobre el nivel del mar, en la propiedad del duque de Ciudad Rodrigo, frente a la Sierra de Parapanda y en línea con el Cerro del Morrón, a 2 kms. de Íllora y a igual distancia de Alomartes, a $3^{\circ} 53^{\prime}$ $4^{\prime \prime}$ de longitud y $37^{\circ} 16^{\prime} 4^{\prime \prime}$ de latitud. Parece un pequeño poblado, que en un momento dado se fortifica con un foso y pequeña muralla. Este punto enlaza la fortaleza de Íllora y la torre del Morrón, al tiempo que controla la zona del Molino del Rey y la entrada de la Vega de Granada. Entre los hallazgos hay puntas de flecha de hierro de pequeño tamaño. El carácter del yacimiento no está claro.

\section{0.- Torre de Pedrizaguilla (íllora)}

Situada en el Cerro de Pedrizaguilla, a 92। m. de altura, cerca del Cortijo de los Cortijuelos, A $3^{\circ} 42^{\prime} 22^{\prime \prime}$ y a $37^{\circ} 23^{\prime} 58^{\prime \prime}$ de latitud. Fue la torre mejor situada para vigilar la Vega de Granada, de Obeilar a Sierra Elvira. Tenía visión directa con la fortaleza de Íllora, y las torres siguientes: hacia el norte, Torre de La Mesa, Torre de la Gallina, y Torre del Morrón, y hacia el Oeste, El Fuerte. Sus restos se desmontaron en los años setenta para la explotación de una cantera. Se la menciona en las crónicas de la toma del castillo de íllora.

\section{LÍNEA TOCÓN-RÍO GENIL}

Tocón es el punto de confluencia de los grupos de torres que rodean íllora y Montefrío respectivamente. A partir de ella puede distinguirse una línea interior de torres que primero llega hasta el río Genil. La mayoría de ellas son torres de alquería, es decir, están enclavadas en las proximidades de localidades, o en estas mismas, teniendo como función servir de refugio a la población del entorno.

\section{I.- Torre de Clementino (Tocón)}

Situada en la localidad de Tocón. Figura en el Inventario de Monumentos de Arquitectura Militar del Patrimonio Histórico Artístico como Torre Clementino, y fechada en el siglo XIV. Situada al Sudoeste de la
Sierra de Parapanda, a $3 \mathrm{kms}$. de la torre de la Encantada y a 10 de la fortaleza de Íllora, a $3^{\circ} 57^{\prime} 5$ I' de longitud y $37^{\circ} 14^{\prime} 2 l^{\prime \prime}$ de latitud, tenía visión directa con las torres del Morrón y la misma de la Encantada. Es de planta cuadrada, con tres plantas abovedadas; las esquinas estaban reforzadas con sillares. A causa de las reformas realizadas por los propietarios de la misma, carece de escaleras, habiendo incorporado la primera planta a una vivienda. La zona cercana a la torre es conocida como el "barrio moro", y de él proceden cerámicas y monedas; asimismo se ha localizado una necrópolis. Es una clara torre de alquería (Lám. 8).

\section{2.- Torre de La Encantada. (Brácana-Íllora)}

Está situada a $2 \mathrm{kms}$. de la población de Brácana y a 3 kms. de la de Tocón, dentro del término municipal de Íllora; a $3^{\circ}$ 57' 5 I" de longitud y $37^{\circ} 13^{\prime} 25^{\prime \prime}$ de latitud. Con una altura de $639 \mathrm{~m}$. sobre el nivel del mar, esta torre controlaba una amplia franja en dirección a Loja y a Alomartes, manteniendo visión directa con la torre de Tocón, y con la del Morrón. La planta es octogonal, con una media de $2 \mathrm{~m}$. de lado y 4,10 m. de anchura máxima. Presenta un sistema de habitaciones parecido al de la torre de Tocón, también abovedadas. En la actualidad sólo se mantienen en pie 3,20 $\mathrm{m}$. de altura. La estructura parece corresponder también a una torre de alquería.

\section{3.- Cerro Vacas (íllora)}

En el límite del término de Íllora, a pocos metros del término de Moraleda de Zafayona, al otro lado del río Genil, a $617 \mathrm{~m}$. de altitud, a $3^{\circ} 56^{\prime} 27^{\prime \prime}$ de longitud y $37^{\circ}$ II' $58^{\prime \prime}$ ' de latitud, se encuentra un cerro en forma de sombrero denominado Cerro Vacas, de 0.2 hectáreas, en el que son visibles los restos de una construcción, posiblemente una torre cuadrada y algunas líneas de piedra, tejas y argamasa, en todo el cerro y en la parte baja, así como abundante cerámica en superficie. Hay otros restos a pocos metros en el término de lillora, en el cerro de enfrente, que parecen ser de menor importancia. Lugar privilegiado para vigilar la vega hacia Loja y hacia Granada. Siendo visibles en su día las torres de La Encantada y del Morrón. 


\section{LÍNEA ZAGRA-LOJA}

Al Oeste del grupo de fortaleza y torres de Montefrío parece existir un cierto vacío, aunque lo agreste del terreno hizo que la zona estuviese lejos de ser un pasillo hacia el interior de la vega. El siguiente punto fuerte de la frontera lo constituia el conjunto formado por la fortaleza adelantada de ZAGRA, con la muy importante de LOJA situada en profundidad (Malpica 1987). Se trata hasta cierto punto de una línea simétrica a la que desde Montefrío llegaba al Genil y que parece haber formado una línea que protegía el flanco noroccidental de la Vega de Granada.

\section{4.- Torre Pesquera (Zagra)}

Pequeña fortificación situada a pocos metros del cauce del río Pesquera, a 200 m. de la carretera que une a Zagra con Fuentes de Cesna y a $4 \mathrm{kms}$. de la fortaleza de Zagra, en dirección noreste. Longitud $4^{\circ}$ II' $24^{\prime \prime}$ y $37^{\circ}$ 17' 15" de latitud. El lugar forma un tajo sobre el río, quedando en pie una torre de $9 \mathrm{~m}$. de altura, de forma circular, unida a otras construcciones donde son visibles parte de una habitación y otros restos. Su construcción es de mampostería. Los restos visibles ocupan una extensión de $50 \mathrm{~m}$. de largo por $10 \mathrm{~m}$. de ancho. También hay abundante cerámica esparcida por toda la ladera sur. Perfectamente situada para controlar toda la cuenca del río desde Algarinejo en dirección sur, y proteger las huertas situadas a ambos márgenes del río, así como una vieja presa, resto del sistema de riego de las huertas, posiblemente de época árabe.

\section{5.- Torre Martilla (Zagra)}

Situada en Santa Barbara, a 799 m. de altu$\mathrm{ra}$, a $4^{\circ} 10^{\prime} 40^{\prime \prime}$ de longitud y $37^{\circ} 14^{\prime} 00^{\prime \prime}$ de latitud, a 3 kms. de Zagra y 20 kms. de Loja. Toma su nombre del cortijo y tierras cercanas. Conserva 2,50 m. de altura, muy deteriorada. Es de planta redonda y en su construcción se observan restos de materiales de un poblado visigodo y una necrópolis, que ocupa gran parte del cerro donde se encuentra situada esta torre. Vigilaba las tierras y el valle entre Loja y Zagra, fortalezas con las que tenía visión directa, dominando también una amplia zona en dirección a Iznájar (Lám. 9).

\section{6.- Torre Agicampe (Loja)}

Cerca de la Loma del Pino se encuentra esta torre, situada a 3,5 kms. de la carretera de Loja a Zagra, a 5,5 kms. de la primera, y a 4 kms. de Huétor Tajar, en el alto del Arroyo de Guantero a $700 \mathrm{~m}$. de altura sobre el nivel del mar. A $4^{\circ} 05^{\prime} 55^{\prime \prime}$ de longitud y a $37^{\circ} 12^{\prime} 58^{\prime \prime}$ de latitud. De planta ovalada, construida en mampostería, conserva $8,5 \mathrm{~m}$. de altura. Tiene una ventana orientada hacia el sur (la Vega) construida con piedra de cantería; la puerta se encuentra tapiada, ya que los propietarios han construido cuadras para animales alrededor de la misma. Consta de dos grandes plantas, la baja utilizada hasta hace poco tiempo para albergue de animales. En la actualidad todos los techos están desplomados. En la parte este y sur de la torre encontramos gran cantidad de cerámica islámica así como restos de construcciones, el asentamiento debía tener I hectárea. Esta torre está construida junto a un gran nacimiento de aguas que hace poco tiempo regaba más de cien hectáreas de terreno. En la actualidad este agua es la que abastece al pueblo de Huétor Tájar (Lám. 10).

\section{7.- Torre de la Torrecilla (Loja)}

Cota $872 \mathrm{~m}$. de altura, a I,5 kms. del Cortijo de la Toba; a 6 kms. de Loja y a 2 kms. de la Venta del Rayo, en la antigua carretera de Málaga. A $4^{\circ}$ II' $56^{\prime \prime}$ de longitud y $37^{\circ} 08^{\prime}$ 03" de latitud. Con una altura de $2 \mathrm{~m}$. en la cara oeste y I,50 m. en la norte. Es de planta redonda; construida de mampostería de grandes proporciones. Posiblemente tuvo una habitación. Toda la franja situada al oeste, entre Archidona e Iznájar, queda bajo el control de este punto de vigilancia. Visibles las torres la Silla del Moro y la del Cortijo del Aire.

\section{8.- Torre del Cortijo del Aire (Loja)}

Situada a $606 \mathrm{~m}$. de altura, al noroeste de la fortaleza, a $500 \mathrm{~m}$. del Cortijo del Aire y a 5 kms. de Loja. A $4^{\circ}$ II' 59" de longitud y a $37^{\circ}$ II' 17" de latitud. Situada en un cerro cónico, al borde del río Genil, que tiene una extensión de cuatro hectáreas, ocupadas casi en su totalidad por los restos de un oppidum 
ibérico; el lugar continuó habitado en época romana e islámica. La torre es de planta redonda y conserva una altura de $2 \mathrm{~m}$. en la cara este. Vigilaba las cuencas del río y otros puntos oscuros hacia el oeste. Tenía visión directa con la fortaleza, y el resto de las torres de Loja que hemos descrito, así como con la Torre Martilla (Zagra).

\section{9.- Torre de la Silla del Moro (Loja)}

Situada a $587 \mathrm{~m}$. de altura, a 2 kms. del Cortijo Garcés, y a $7 \mathrm{kms}$. de la fortaleza de Loja, en el margen izquierdo de la carretera de Loja a Iznájar, a 2 kms. de Ventas de Santa Bárbara. Cerca del lugar hay minas de minio. A $4^{\circ}$ II' 58" de longitud y a $37^{\circ} 10^{\prime} 45^{\prime \prime}$ de latitud. De planta redonda y una altura en la parte sur de $3 \mathrm{~m}$., estando muy deteriorada en la parte norte. Construida de mampostería. Tenía visión directa con la fortaleza de Loja, y con las torres de La Torrecilla, Cortijo del aire, hacia el noroeste, y con la de la Majada de Cobo en la Sierra de Loja hacia el sureste. Vigilaba, hacia el sur, los puntos oscuros, y los límites con Iznájar, y la sierra cercana a Archidona.

\section{LÍNEA EL SALAR-ALHAMA DE GRANADA}

Al Sur del río Genil, siguiendo aproximadamente el recorrido del río de Alhama que desemboca en el primero, se encuentran diversas torres, que en cierta forma podría considerarse que forman otra línea que continúa la de Tocón-Río Genil, pero que a su vez conecta con las torres de Loja. Como muchas de las que hemos visto con anterioridad, la mayoría son torres alquería.

\section{0.- Torreón de Los Tajos (Salar)}

Situado a 2.500 m. de El Salar, en el km. 12 que une la carretera de Salar a Alhama, en el cerro del mismo nombre a $743 \mathrm{~m}$. altura. A $4^{\circ} 04^{\prime}$ I I" de longitud y $37^{\circ} 08^{\prime}$ ' $22^{\prime \prime}$ de latitud. La base que quedaba de esta torre fue desmontada hace unos años, quedan esparcidos por el lugar restos de cerámica. Esta torre controlaba las zonas oscuras que van desde el Salar a la torre de la Gallina.

\section{I.- Torre de La Gallina (Salar)}

Se encuentra situada a $984 \mathrm{~m}$. altura, en el $\mathrm{km}$. I 8 que une la población de Alhama con el Salar, en el límite de ambos municipios. A $4^{\circ} 0 l^{\prime} 29^{\prime \prime}$ de longitud y $37^{\circ} 05^{\prime} 22^{\prime \prime}$ de latitud. Torre de mampostería, conserva I,5 m. de altura, estando muy deteriorada. Por la cantidad de material acumulado a su alrededor no se aprecia si es cilíndrica o cuadrada. En la actualidad presenta una pequeña "cueva", por lo que se supone que en origen no fue maciza. Esta torre enlazaría con la torre de Buenavista y controlaría las tierras y puntos oscuros hasta la siguiente torre, la denominada Torre de La Solana.

\section{2.- Torre de La Solana (Alhama de Granada)}

Situada a 3.500 m. de Alhama de Granada en el paraje conocido como Cortijo Rodero. A $3^{\circ} 59^{\prime} 29^{\prime \prime}$ de longitud y $37^{\circ}$ Ol' 22 de latitud. Es de planta redonda, construida en mamposteria, de grandes dimensiones, conservando 10 m. de altura, debía tener al menos tres plantas, aunque hoy todas tienen los techos desprendidos. Próximos a la torre hay otros restos de construcciones, lo que confirma la existencia de un hábitat en torno, debía tratarse de una alquería. Controlaba las tierras que van desde Alhama a la torre de Agrón y las que hacia el norte enlazarían con la Torre de Buenavista cerca de Santa Cruz del Comercio (Lám. II).

\section{3.- Torre de Buenavista (Alhama de Granada)}

Situada a $10 \mathrm{kms}$. de Alhama de Granada y a otros tantos de Moraleda de Zafayona, a $984 \mathrm{~m}$. de altura. A $3^{\circ} 58^{\prime} / 7^{\prime \prime}$ de longitud y $37^{\circ} 14^{\prime} 40^{\prime \prime}$ de latitud. Es maciza, de mampostería, de planta octogonal con una altura de 3, 10 metros. Tenía buena visibilidad de las tierras que unen las torres de la Gallina y la Solana, así como la Atalaya de Agrón hacia noroeste, dominando una amplia extensión de vega (Lám. 12).

\section{LÍNEA ALHAMA-ZAFARRAYA}

Esta zona tenía gran importancia, ya que es el paso principal entre la vega de Granada y la costa malagueña. Por el momento sólo conocemos una torre, aunque es muy posible 
que existiesen otras dos hoy desaparecidas que completarían el sistema.

\section{4.- ¿Torre Donna?}

La primera de las torres posibles se situaría al oeste de la fortaleza de Alhama de Granada, en el lugar conocido como torre Donna, y cerca del cortijo del mismo nombre, y que enlazaría la fortaleza de Alhama con la torre de La Torrecilla.

\section{5.- La Torrecilla (Alhama de Granada)}

Situada a 15 kms. de Alhama. A 4 04' I I" de longitud y a $36^{\circ} 58^{\prime} 2$ I" de latitud, a 132 I m. de altura. De construcción maciza, en mampostería, y planta circular, con una altura en la cara norte de $40 \mathrm{~cm}$., hay otros restos adosados a la torre. Controlaría las tierras al sur de Alhama de Granada en la Sierra de Alhama y el boquete de Zafarraya.

\section{6.- iTorre Jota?}

Para completar el sistema cabe la posibilidad de que existiera otra torre entre Alhama y Zafarraya a continuación de la torrecilla en el lugar conocido por Torre Jota (T. Blanca) a 5 kms. de Zafarraya.

\section{LA VEGA EN EL ENTORNO DE GRA- NADA}

\section{Flanco Norte}

En la parte norte de la Vega de Granada, y de Este a Oeste se encuentran las torres próximas a las localidades de Cogollos Vega, Alobolote, Chaparral de Cartuja y Pinos Puente.

\section{7.- Torre Atalaya (Cogollos Vega)}

Se encuentra cerca del collado de la Cruz de la Atalaya, a I.I56 m. de altura sobre el nivel del mar, a $7 \mathrm{kms}$. de la población de Deifontes y a 5 kms. de Cogollos. A $3^{\circ} 34^{\prime}$ I I" de longitud y 370 18' 36" de latitud. Controlaba las tierras y barrancos completando el control hacia el norte de todas las tierras en dirección a Iznalloz, contando para completar esta franja de terreno con las torres del Chaparral de Cartuja y la torre de Albolote en el oeste. Construida en mampos- tería. Es la mejor conservada quizás de todo el sistema, salvo por un agujero en su base en la parte norte. Maciza hasta la mitad, con una habitación a $5 \mathrm{~m}$. de altura, con dos ventanas, una en la parte norte y otra orientada hacia el sur; conserva el techo de esta habitación; su altura es de ocho metros.

\section{8.- Torre del Chaparral de Cartuja (Güevéjar)}

Situada a 5 km. de Güevéjar, a 899 m. de altura, entre los cortijos el Chaparral de Cartuja al oeste y el Regajo al este, a 400 m. de la carretera que va a Güevejar. A $3^{\circ} 36^{\prime}$ 52 " de longitud y $37^{\circ}$ 15' 2 I'" de latitud. Es de planta redonda, desmoronada. Conserva una altura de 80 cms. Su construcción es de mampostería. Completaba junto a la torre de Albolote, Cogollos y el Castillejo el control de las tierras situadas al norte de la Alhambra, y el valle que va desde Iznalloz al río cubillas. El Castillejo, a l.085 metros de altura, al que nos hemos referido, está al noreste de Güevéjar, y es un lugar en forma de pequeña meseta rodeada de tajos entre Güevéjar y Nívar.

\section{9.- Torre de la Atalaya (Albolote)}

Cerca del Collado del Pino, a $885 \mathrm{~m}$. de altura sobre el nivel del mar, a 4 kms. de la población de Albolote, frente a la torre cercana al Chaparral de Cartuja, hacia el norte a 5 km. queda el Pantano de Cubillas. A $3^{\circ} 40^{\prime}$ $55^{\prime \prime}$ de longitud y $37^{\circ}$ 14' 47" de latitud. Su construcción es de mampostería, con dos ventanas en la parte superior de la torre. La mitad inferior es maciza, de idénticas características a la torre de Cogollos Vega y a las cercanas a Moclín. Su altura es de 9 m. En la parte norte amenaza derrumbarse toda la zona que forma la ventana. Hay restos de una construcción junto a la base de la torre, con varias líneas de viviendas. Son visibles desde éste punto hacia el norte el Pantano de Cubillas y la zona del Chaparral, hacia el noroeste la fortaleza de Moclín y hacia el sur Romilla y Santa Fe (Lám. 13).

\section{0.- Peñón de Zujaira (Pinos Puente)}

Situado a $500 \mathrm{~m}$. al noroeste de la población del mismo nombre, junto al barranco del Peñón en el km. 6 de la carretera de íllora a Zujaira. A $3^{\circ} 48^{\prime} 22^{\prime \prime}$ de longitud y $37^{\circ}$ I5' 
49" de latitud. La altura de la roca, 17 metros, fue utilizada como puesto de vigilancia, y a su alrededor se extendía una población, como indica la cerámica esparcida por el terreno en una extensión de 1,5 hectáreas. Este sería posiblemente el asentamiento árabe destruido que nos narran los cronistas de la conquista de Granada. En el barranco cercano al lugar, en la cantera, hay tumbas tardorromanas excavadas en la roca.

\section{Flanco Occidental}

\section{I.-Torre de Romilla. (Granada)}

A 300 m. de la población de Romilla, cerca del río Genil, y a 3,5 kms. de Santa Fe, se encuentra esta torre de grandes proporciones, de tres plantas, de características similares a algunas torres que se encuentran adosadas a la muralla de la Alhambra. A $3^{\circ} 47^{\prime} 40^{\prime \prime}$ de longitud y a $37^{\circ} 12^{\prime} 34^{\prime \prime}$ de latitud. Cuenta con varias aspilleras y ventanas; en las caras Oeste y Este hay dos aspilleras en la segunda planta, y una ventana en la tercera. En las caras Norte y Sur, sólo una aspillera en el segundo piso y una ventana en el tercero. En el lado Este está la puerta, a $2 \mathrm{~m}$. de la base. Las escaleras y los techos de las tres plantas están desmoronados, así como la puerta. Las dimensiones en su base son: $14,30 \mathrm{~m}$. de lado oeste y $7,26 \mathrm{~m}$. sur y norte, altura aproximada 12 metros. Está construida de mampostería. El ladrillo se emplea en las puertas, ventanas y escaleras. La torre de Romilla, cerca del río Genil, puede considerarse el final del flanco norte, ya que en cierta forma dobla la de Pinos Puente, situada al Norte, casi en la misma línea. El resto se encuentran al Sur del río Genil. De Norte a Sur son las de Boldonar, La Malaha y la de Agrón, aunque sin formar una línea.

\section{2.- Atalaya de Boldonar (Cerro Alonso)}

A 4,5 kms. de la población de Peñuelas, a 2 kms. del pueblo de Chimeneas, a $675 \mathrm{~m}$. de altura sobre el nivel del mar, en la parte éste del Cerro Alonso y del Arroyo de Chimeneas. Se encuentra esta torre (atalaya) a $9 \mathrm{kms}$. del aeropuerto de Granada, a pocos metros de la cota Boldonar. A $3^{\circ} 50^{\prime} 20^{\prime \prime}$ de longitud y $37^{\circ} 09^{\prime}$ 19" de latitud. Es una de las más pequeñas estudiadas, de planta cuadrada con $4 \mathrm{~m}$. de lado, se consevan unos $7 \mathrm{~m}$. de altura en la cara oeste; tenía tres plantas, siendo aún visibles las lineas de separación de la primera y segunda planta. Su construcción es de tapial, con una base de ladrillos y mampostería utilizada en la separación de cada una de las plantas. Esparcidos por el olivar cercano a la torre se encuentran gran cantidad de cascajo y cerámica árabe en una extensión de una hectárea. Torres visibles desde este punto: La Malahá, Romilla, y a mayor distancia la Atalaya de Agrón (Lam I5).

\section{3.- Atalaya de la Malahá}

Situada en la cota del mismo nombre a $883 \mathrm{~m}$. de altura sobre el nivel del mar, en el paso conocido como Chinchilla, a 2,5 kms. de la población de la Malahá. A $3^{\circ} 44^{\prime}$ I 3 " de longitud y $37^{\circ} 06^{\prime} 39$ de latitud. Totalmente destruida. Solamente se aprecia la elevación de sus restos en forma de cono. Hay restos de cerámica y cascajo alrededor de la torre. Salinas y un aljibe a pocos kms. de la torre. Vigilaba los Ilanos de Escúzar, Ventas de Huelma y El Padul. Las torres atalaya más cercanas a ésta son al oeste la del Boldonar, cerca de Chimeneas, y al sur la Atalaya de Agrón;

\section{4.- Atalaya de Agrón.}

Fuera de la línea anterior, a medio camino de esta zona y la ciudad de Granada, en el flanco Suroeste de la ciudad, se encuentra la localidad de Agrón, que da nombre a una torre situada en las proximidades. Está a $1.168 \mathrm{~m}$. de altura sobre el nivel del mar, frente al pantano de los Bermejales, a $3^{\circ} 57^{\prime} 44^{\prime \prime}$ de longitud y $37^{\circ}$ OI' I $3^{\prime \prime}$ de latitud. A 14 $\mathrm{kms}$. al Oeste queda la fortaleza de Alhama de Granada, y a 4 kms. Agrón, cerca del Barranco de las Rejas. Esta torre, situada el este de la fortaleza, controlaba hacia el sur las tierras de Alhama y hacia el este Ventas de Huelma en dirección a Escúzar. Es de planta octogonal, maciza, construida con mampostería, de idénticas características a las de la torre de La Encantada (Íllora); relativamente conservada en el lado norte, donde tiene $2 \mathrm{~m}$. de altura, bastante deteriorada el lado sur. Con hábitat en torno. A $2 \mathrm{~m}$. de la base se distingue un pequeño estanque, actualmente relleno, y muy difícil de fechar por el momento. 


\section{EL SISTEMA INTERNO TRANSVERSAL}

Al igual que la distribución de las torres de Norte a Sur pueden considerarse como pertenecientes diversas líneas que protegerían en profundidad la parte Occidental de La Vega. A modo de hipótesis apuntamos que algunas de ellas pudieron construirse, al menos en parte, para formar un segundo cinturón de torres transversal en la parte Norte de la Vega, que controlarían el territorio existente entre las fortalezas "exteriores" y las torres próximas a la propia Granada.

Este cinturón partiría de la TORRE DE AGICAMPE, que tenía visión directa con la TORRE DEL AMARGUILLO (desaparecida), situada en el Cerro de la Torre, a 3 kms. de Villanueva de Mesías. Esta enlazaba con la Torre de LA ENCANTADA (Brácana), la cual contaría con otro punto de apoyo y vigilancia en el lugar conocido como CERRO VACAS.

Desde este punto se tiene visión directa hacia el Norte con EL MORRÓN de Parapanda, con LA ENCANTADA y a mayor distancia con EL FUERTE, y ésta a su vez con la torre de CLEMENTINO (Tocón).

A continuación el punto conocido como El Fuerte (TORRE DE LOS INGLESES) a $816 \mathrm{~m}$. de altura y muy cerca de este punto anotamos también otra torre desaparecida en La loma de Taura en el lugar denominado LA ATALAYA, a $2 \mathrm{kms}$. de esta aldea. Le seguiría con visión directa la torre de PEDRIZAGUILLA, en la cota 82I, cerca del cortijo de Los Cortijuelos, a 3 kilómetros de íllora (desaparecida). A continuación el PEÑÓN DE ZUJAI$R A$, que aunque no presenta restos concretos de torre, en torno al mismo se extendía un amplio hábitat de I ha. siendo la cresta del peñón una evidente atalaya natural.

Después la TORRE de Pinos Puente, localidad fundamental para acceder a Granada, bien conocida por su puente, construido en época emiral. A continuación el ALTO DE SIERRA ELVIRA, en la cota 828, otro lugar natural de observación, donde existe cerámi- ca en superficie. El punto siguiente sería la ATALAYA de Albolote y con esta última enlazarían las situadas hacia el Este, torres del CHAPARRAL DE CARTUJA y de COGOLLOS VEGA, que tenían visión directa con la Alhambra de Granada.

Este sería el segundo cinturón de torres y puntos de vigilancia al filo de la Vega de Granada, en muchos de los cuales hay abundantes restos de cerámica que indican la existencia de poblamiento en torno a las mismas, que posiblemente deben identificarse con algunas de las numerosas alquerías citadas por Ibn al-Jatib. La construccción de estas torres en puntos no demasiado elevados, respondería en parte a la necesidad de enlazar las torres fronterizas con el área interior, dado que en invierno las torres más lejanas, como por ejemplo la situada en el Morrón de Sierra de Parapanda, no servirían de nada, porque las nieblas que ocupan zonas de la Vega impedirían su visión.

\section{BIBLIOGRAFÍA}

ARGUELLES MÁRQUEZ, M. (1987): "El sistema defensivo Montefrío-Moclín en época nazari". II Congreso de Arqueología Medieval Española (Madrid 1987). Madrid pp. 85-9l.

CRESSIER, P. (1984): "Las fortalezas musulmanas de la Alpujarra (Provincias de Granada y Almería) y la división político-administrativa de la Andalucía oriental". Arqueología Espacial, Vol. 5, pp. 179-199.

CRESSIER, P. (1992): "El castillo y la división territorial en la Alpujarra medieval: del hisn a la taca" en CRESSIER, P. et alii: Estudios de arqueología medieval en Almería. Almería pp. 7-48.

GUICHARD, P. (1986): "Perspectives de recherche sur la toponymie et la géographie historique d'al-Andalus oriental" en BAZZANA, A. y POISSON, J. M. (eds.): Histoire et archéologie de l'habitat médiéval. Lyon, pp. 185-190.

MALPICA CUELLO, A. (1987): "El castillo de Zagra y el alfoz de Loja a fines de la Edad Media". Homenaje al profesor Juan Torres Fontes. Universidad de Murcia, pp. 959-973.

SALVATIERRA CUENCA, V.; ARGUELLES MARQUEZ, M.; MORENO ONORATO, Ma A. (1989): "Visibilidad y control: un problema de fronteras. El caso nazarí en el sector Montefrio-Moclín". Arqueologia Espacial vol. 13, pp. 229-240. 

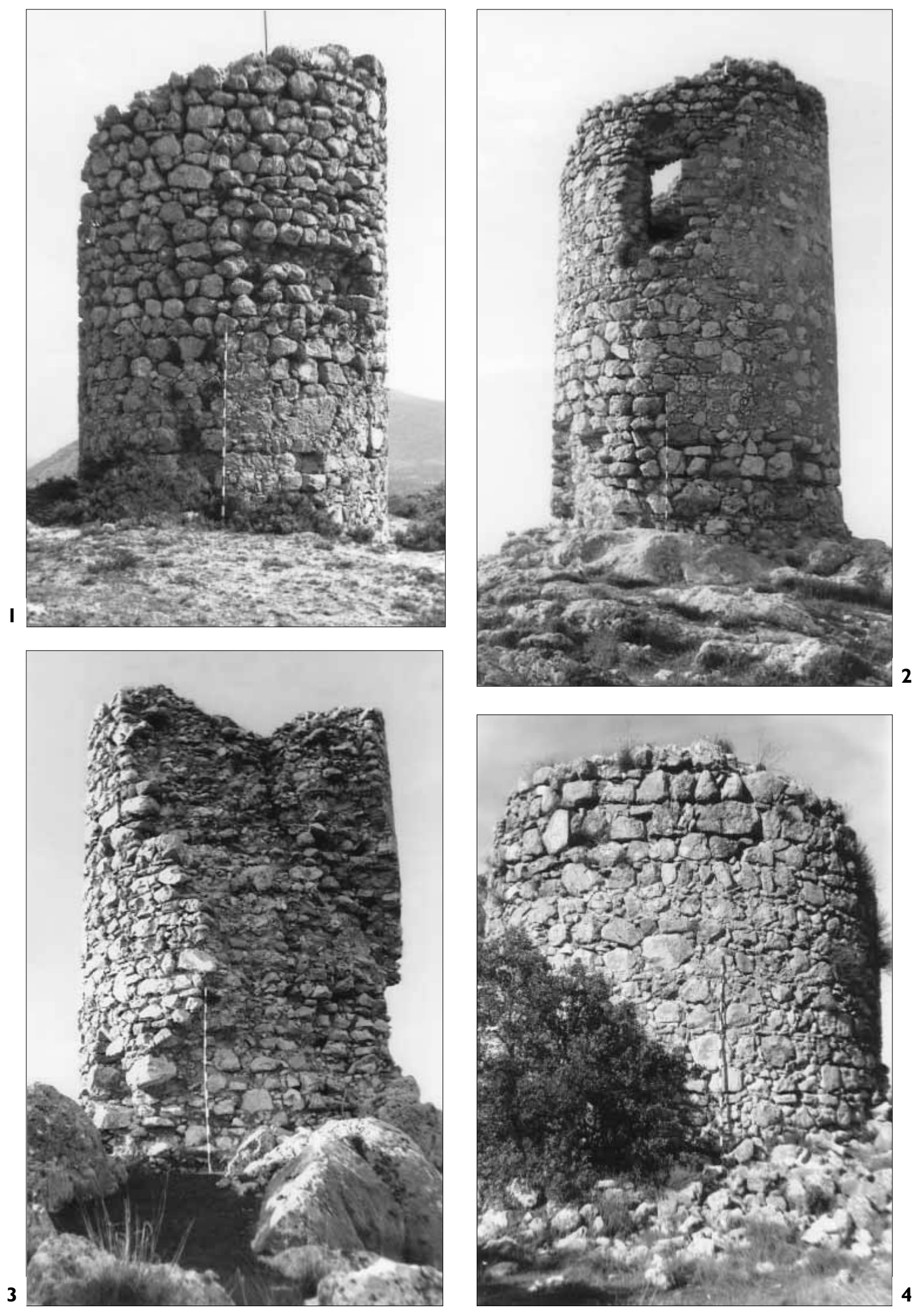

I. Torre de la Atalaya (Colomera) 2. Torre de la Porqueriza (Moclín) $\quad$ 3. Torre de Mingoandrés (Moclín)

4. Torrre de la Mesa (íllora) 

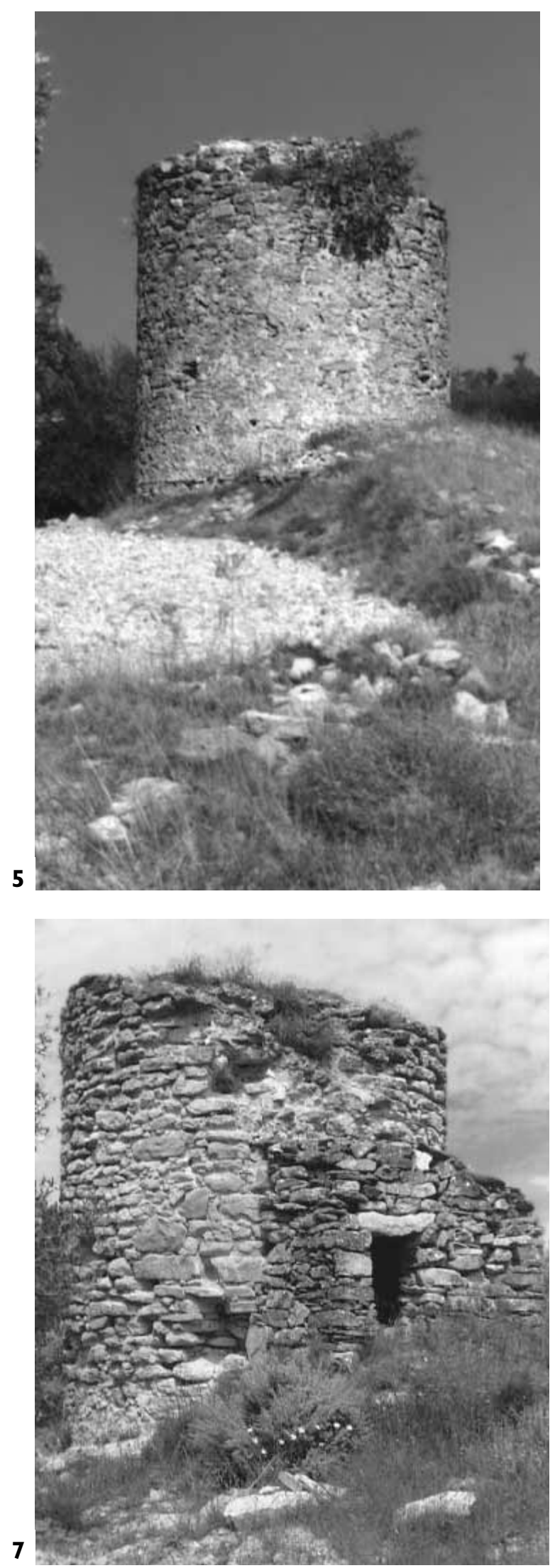
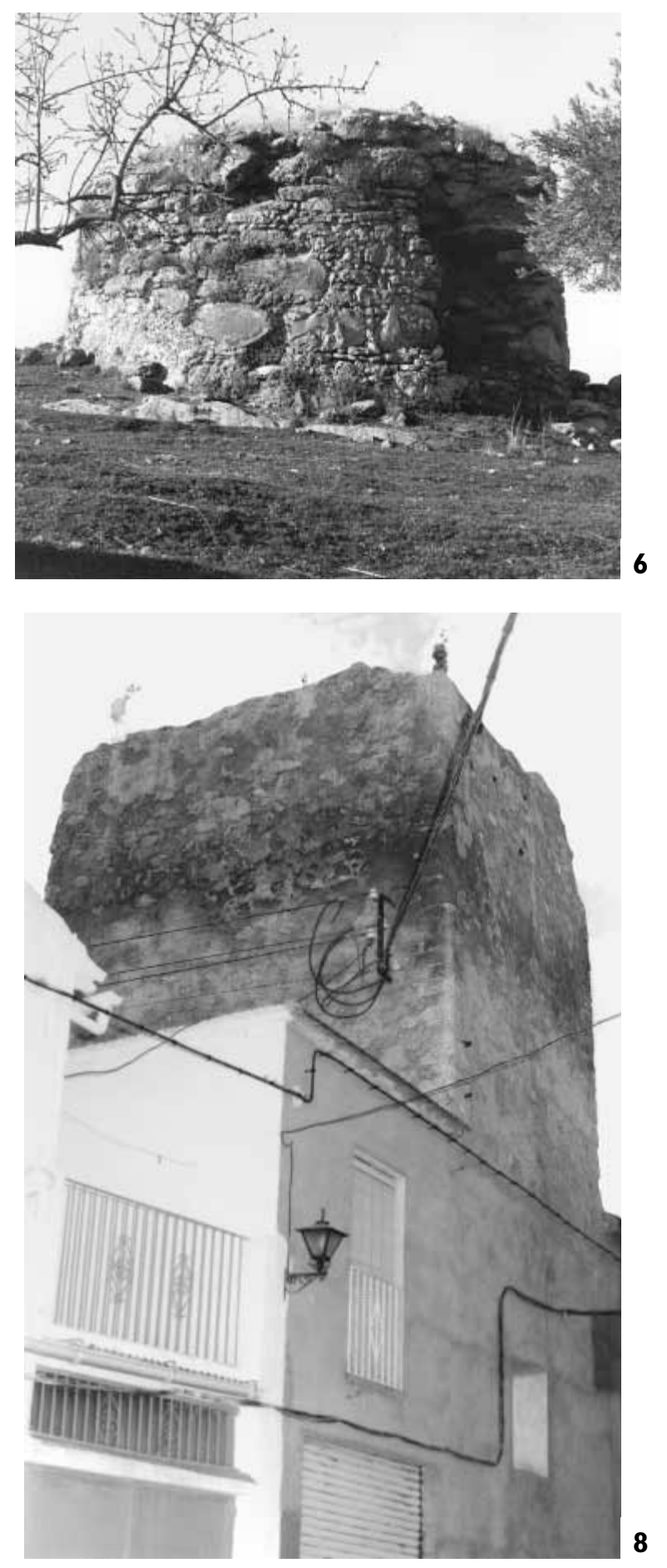

5. Torre del Cortijuelo (Montefrío)

6. Torre de los Anillos (Montefrío)

7. Torre de los Guzmanes(Montefrío)

8. Torrre de Clementino (Tocón) 

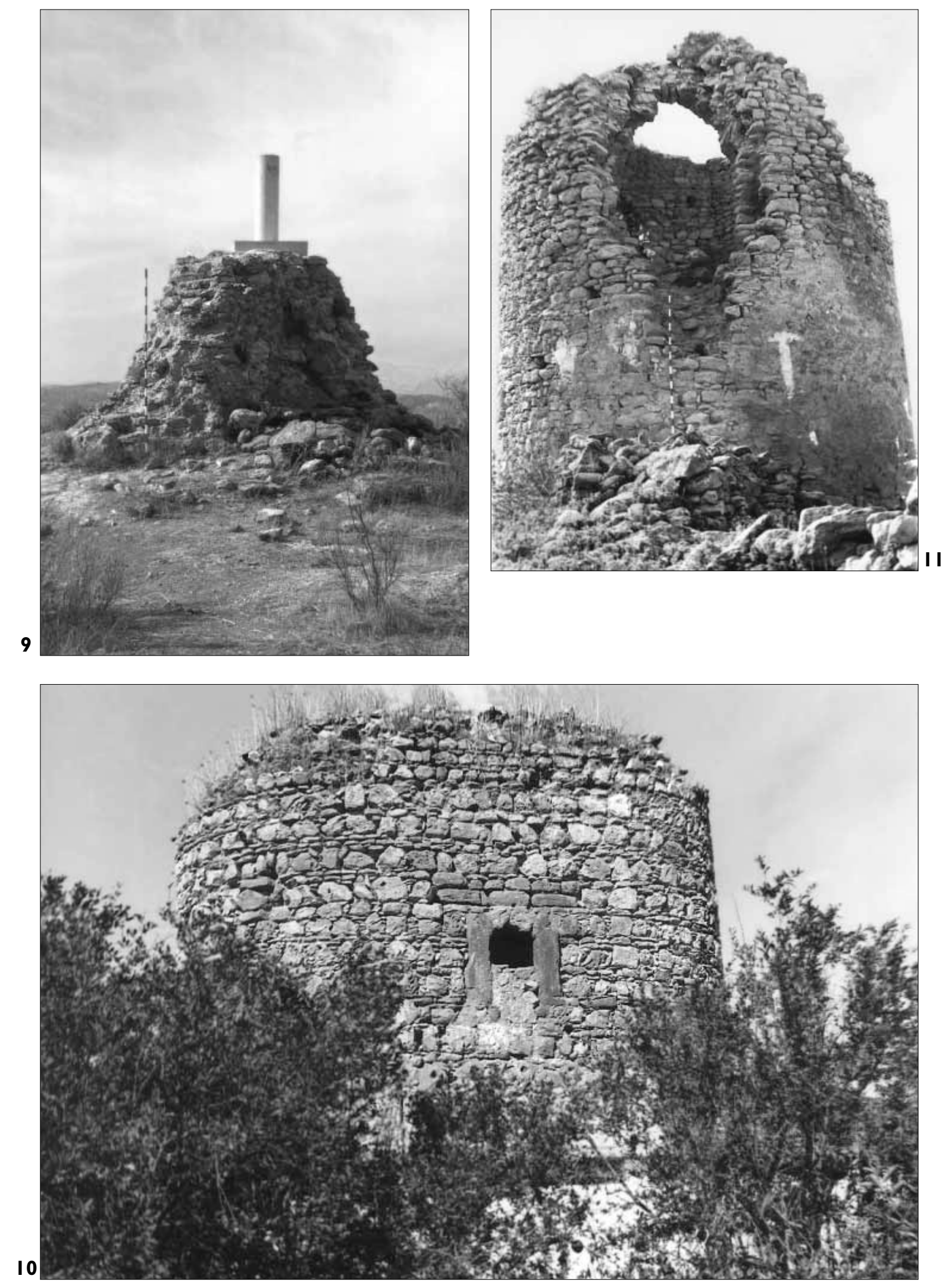

9. Torre Martilla (Zagra)

10. Torre Agicampe (Loja)

II. Torre de la Solana (Alhama de Granada) 

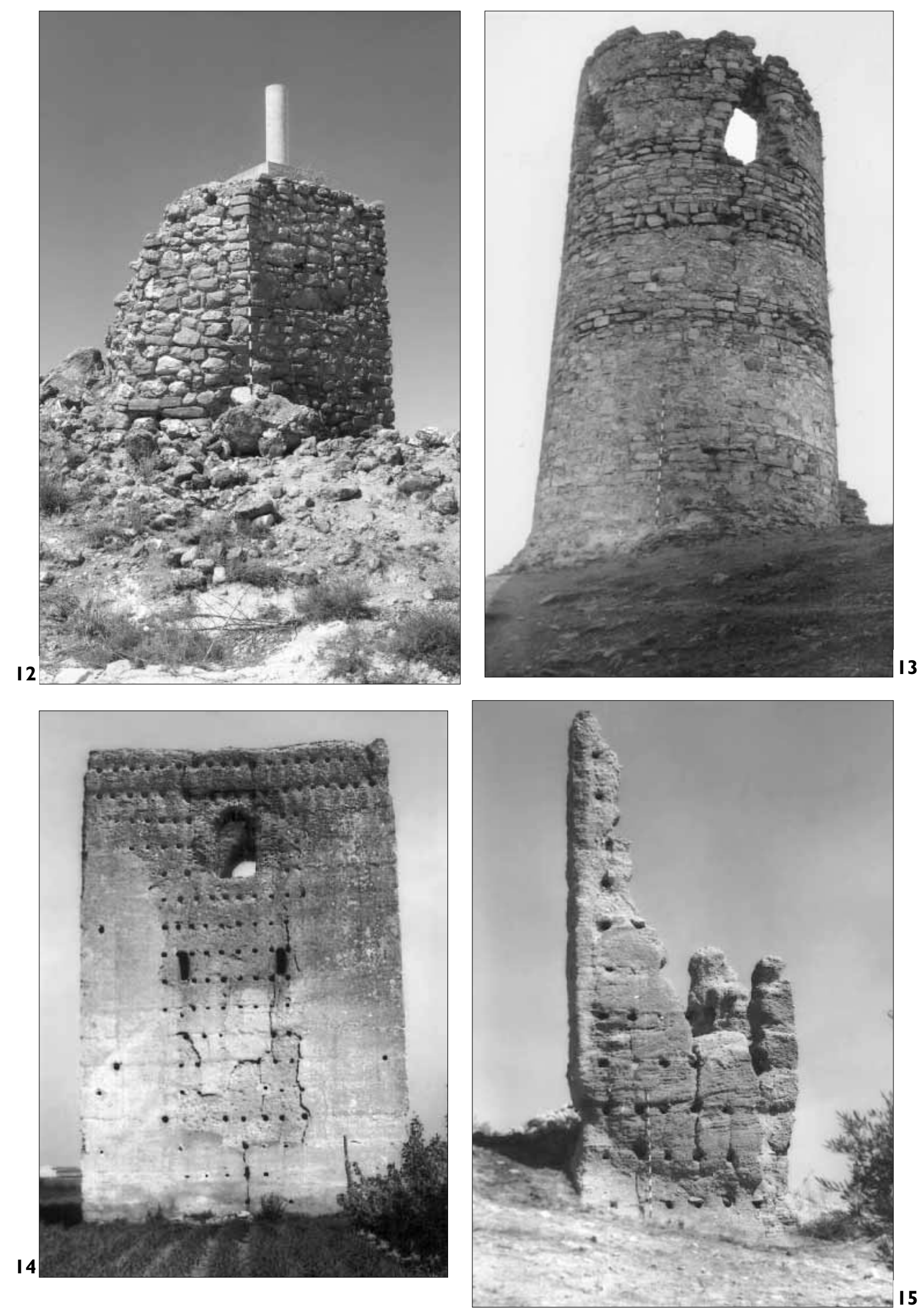

12. Torre de Buenavista (Alhama de Granada)

13. Torre de la Atalaya (Albolote)

14. Torre de Romilla (Granada)

15. Atalaya de Boldonar (Cerro Alonso) 


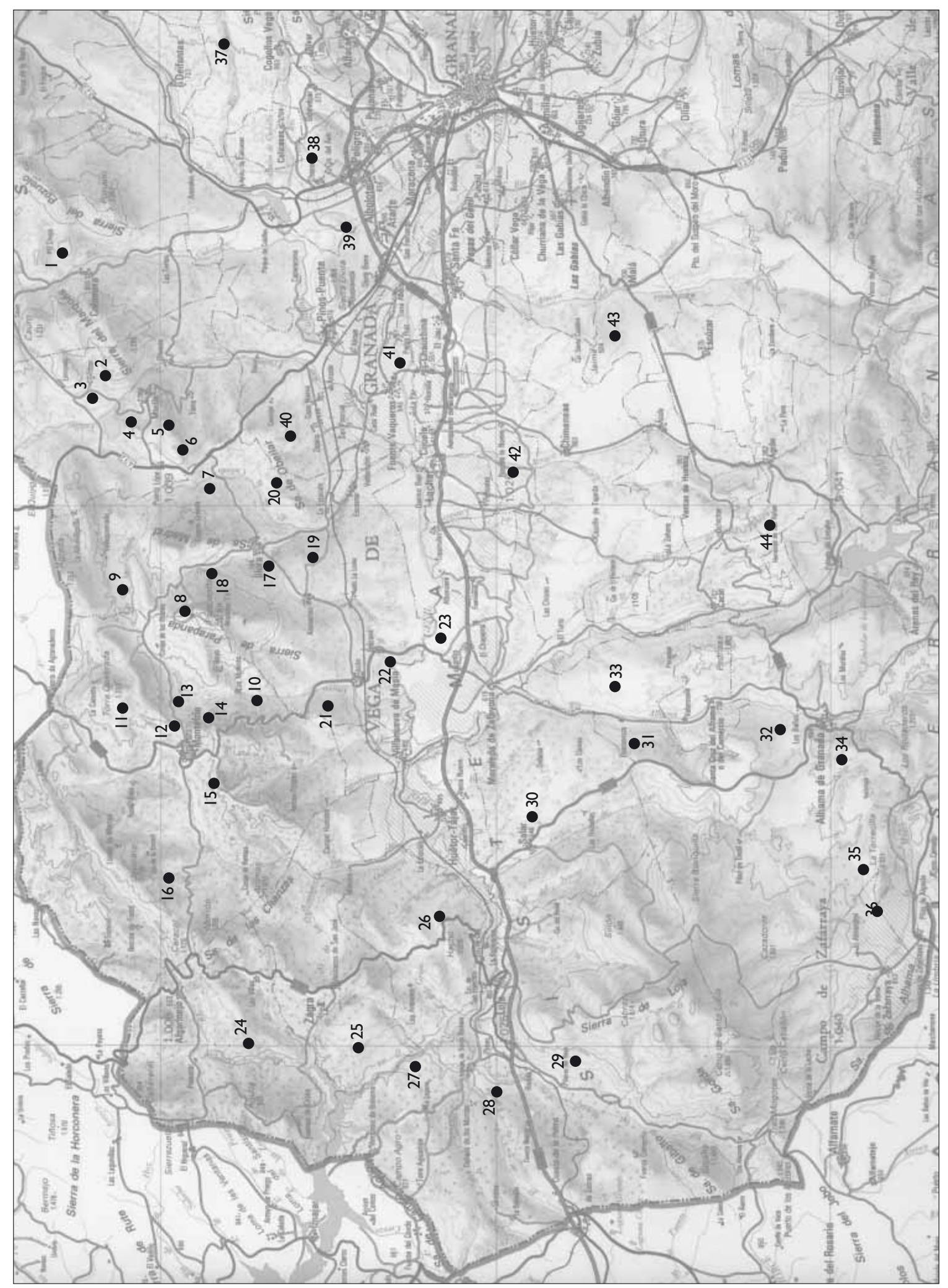

Base del plano: fuente: IGN

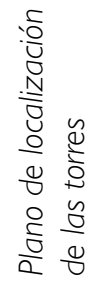

